PROCEEDINGS OF THE

AMERICAN MATHEMATICAL SOCIETY

Volume 126, Number 2, February 1998, Pages 405-409

S $0002-9939(98) 04115-\mathrm{X}$

\title{
NON-ANALYTIC HYPOELLIPTICITY IN THE PRESENCE OF SYMPLECTICITY
}

\author{
NICHOLAS HANGES AND A. ALEXANDROU HIMONAS \\ (Communicated by Jeffrey B. Rauch)
}

\begin{abstract}
Here we construct non-analytic solutions to a class of hypoelliptic operators with symplectic characteristic set and in the form of a sum of squares of real analytic vector fields.
\end{abstract}

\section{INTRODUCTION AND RESULTS}

The purpose of this work is to construct non-analytic solutions to a class of partial differential equations $P u=0$, where $P=X_{1}^{2}+X_{2}^{2}+X_{3}^{2}$, and $X_{1}, X_{2}, X_{3}$ are real vector fields with real-analytic coefficients in $\mathbb{R}^{3}$. All points of $\mathbb{R}^{3}$ are of finite type, and therefore by Hörmander's sum of squares theorem the operator $P$ is hypoelliptic. Also, the characteristic set of $P$ is symplectic, and therefore contains no curves orthogonal to its tangent space with respect to the fundamental symplectic form. This shows that the existence of such curves is not the deciding factor which distinguishes hypoellipticity from analytic hypoellipticity. It also shows that a necessary condition for analytic hypoellipticity, conjectured by Treves [Tr], can not be sufficient. We shall explain all this in more detail after we state the main result.

Theorem 1.1. Let $k$ be an odd positive integer, and $P$ be the operator in $\mathbb{R}^{3}$ defined by

$$
P=\partial_{x_{1}}^{2}+x_{1}^{k-1} \partial_{x_{2}}^{2}+x_{1}^{2 k} \partial_{x_{3}}^{2} .
$$

Then one can construct non-analytic solutions to the equation $P u=0$ near the origin.

Remark. The class of operators in (1.1) is contained in a class studied by Oleinik and Radkevic [OR1]. There, necessary and sufficient conditions for analytic hypoellipticity are given. The existence of singular solutions is proved by indirect methods. The important point of our work is the explicit construction of singular solutions, especially formula (3.1). The construction here falls into the framework of the methods developed in recent years by Helffer [He], Christ [C1], [C2], Hanges and Himonas [HH1], [HH2], Pham The Lai and Robert [PR], and others.

Received by the editors July 22, 1996.

1991 Mathematics Subject Classification. Primary 35H05.

Key words and phrases. Analytic hypoellipticity, sum of squares of vector fields, finite type, characteristic set, symplectic, eigenvalue, eigenfunction.

The first author was partially supported by NSF Grant DMS 91-04569, and the second author by NSF Grant DMS 91-01161.

(C) 1998 American Mathematical Society 
In order to describe the implications of Theorem 1.1 we shall first state the general problem about analytic hypoellipticity of a sum of squares of vector fields. Let $\Omega$ be an open set in $\mathbb{R}^{n}$ and $X=\left\{X_{1}, \cdots, X_{m}\right\}$ be a set of $m$ real vector fields with real analytic coefficients in $\Omega$. We assume that all points of $\Omega$ are of finite type. That is, the Lie algebra of the vector fields $X$ has dimension $n$ at every point of $\Omega$. The generalized Laplacian for the vector fields $X$ is the differential operator

$$
\Delta_{X}=X_{1}^{2}+\cdots+X_{m}^{2} .
$$

This operator is hypoelliptic in $\Omega,[\mathrm{Ho}],[\mathrm{K}],[\mathrm{OR} 2],[\mathrm{RS}]$, although it may not be analytic hypoelliptic [BG]. We recall that an operator $P$ is hypoelliptic in $\Omega$ if for any open subset $U$ of $\Omega$ and for any distribution $u$ in $\mathcal{D}^{\prime}(U)$ the condition $P u \in C^{\infty}(U)$ implies $u \in C^{\infty}(U)$. P is analytic hypoelliptic in $\Omega$ if for any $U$ open in $\Omega$ the conditions $u \in \mathcal{D}^{\prime}(U)$ and $P u$ analytic in $U$ imply $u$ analytic in $U$.

If $(x, \xi)$ are the variables in $T^{*} \Omega$ then the principal symbol of $\Delta_{X}$ is $p(x, \xi)=$ $X_{1}^{2}(x, \xi)+\cdots+X_{m}^{2}(x, \xi)$, and its characteristic set is $\Sigma=\left\{X_{1}(x, \xi)=\cdots=\right.$ $\left.X_{m}(x, \xi)=0\right\}$. We shall assume that $\Sigma$ is a real analytic submanifold of $T^{*} \Omega$. We recall that $\Sigma$ is symplectic if the restriction of the fundamental symplectic form

$$
\sigma=\sum_{j=1}^{n} d \xi_{j} \wedge d x_{j}
$$

to $T \Sigma$ is non-degenerate. It has been proved by Treves [Tr] and Tartakoff $[\mathrm{T}]$, independently, that $\Delta_{X}$ is analytic hypoelliptic in $\Omega$ if $\Sigma$ is symplectic and $p$ vanishes to second order on $\Sigma$. The symplecticity of $\Sigma$ does not allow the existence of Treves curves in it. A non-constant curve $\alpha(t)$ inside the characteristic set $\Sigma$ is said to be a Treves curve for $\Sigma$ if $\dot{\alpha}$ is orthogonal to $T \Sigma$ with respect to $\sigma$ at every point of $\alpha$. That is,

$$
\sigma(\dot{\alpha}, \Theta)=0, \quad \forall \Theta \in T \Sigma, \text { at every point of } \alpha .
$$

If $k=1$ then the operator $P$ in (1.1) is the well known Baouendi-Goulaouic operator which provided the first counterexample to analytic hypoellipticity of a sum of squares operator satisfying the finite type condition [BG]. In this case the principal symbol is $p(x, \xi)=-\left(\xi_{1}^{2}+\xi_{2}^{2}+x_{1}^{2} \xi_{3}^{2}\right)$ and the characteristic set is $\Sigma=\left\{x_{1}=\xi_{1}=\right.$ $\left.\xi_{2}=0\right\}$. Moreover the curve $\alpha(t)=\left(0, t+x_{2}^{0}, x_{3}^{0} ; 0,0, \xi_{3}^{0}\right), \xi_{3}^{0} \neq 0$, is a Treves curve inside $\Sigma$. In fact in all counterexamples to analytic hypoellipticity for the operators $\Delta_{X}$ found in the literature there exists a Treves curve inside their characteristic set, $[\mathrm{BG}],[\mathrm{DZ}],[\mathrm{M}],[\mathrm{PR}],[\mathrm{He}],[\mathrm{HH} 1],[\mathrm{C} 1],[\mathrm{C} 2]$. This is consistent with the following conjecture of Treves $[\mathrm{Tr}]$.

Treves Conjecture. A necessary condition for the analytic hypoellipticity of $\Delta_{X}$ is the following condition $(T)$ :

(T) The characteristic set of $\Delta_{X}$ contains no Treves curves.

Although this conjecture still remains an open problem, Theorem 1.1 implies the following related corollary.

Corollary 1.2. Condition $(T)$ is not sufficient for the analytic hypoellipticity of $\Delta_{X}$.

Proof. If $k=3,5,7, \cdots$, then the characteristic set of $P$ in (1.1) is $\Sigma=\left\{x_{1}=\xi_{1}=\right.$ $0\}$. Since $\Sigma$ is symplectic it does not contain any Treves curves. Since by Theorem $1.1 P$ is not analytic hypoelliptic we conclude that condition $(\mathrm{T})$ is not sufficient, which proves the corollary. 
We mention that Grigis and Sjöstrand [GS] have shown analytic hypoellipticity for a class of operators $\Delta_{X}$ whose characteristic set is not symplectic but it does not contain any Treves curves. And, we conclude this section by stressing the implication of Theorem 1.1 that a hypoelliptic operator $\Delta_{X}$ may not be analytic hypoelliptic and this phenomenon may occur without the presence of Treves curves.

\section{Proof of Theorem 1.1}

First we remark that all solutions to equation $P u=0$ are in $C^{\infty}$. Separation of variables suggests that we should look for non-analytic solutions of the form

$$
u(x)=\int_{0}^{\infty} e^{i \rho^{k+1} x_{3}} e^{\sqrt{\mu} x_{2} \rho^{(k+1) / 2}} A\left(\rho x_{1}\right) w(\rho) d \rho,
$$

where the functions $A$ and $w$, and the positive constant $\mu$ are to be determined. By applying $P$ formally to $u$ we obtain

$$
\begin{aligned}
P u(x)=\int_{0}^{\infty} e^{i \rho^{k+1} x_{3}} e^{\sqrt{\mu} x_{2} \rho^{(k+1) / 2}} & \\
& \times \rho^{2}\left[A^{\prime \prime}\left(\rho x_{1}\right)-\left[\left(x_{1} \rho\right)^{2 k}-\mu\left(x_{1} \rho\right)^{k-1}\right] A\left(\rho x_{1}\right)\right] w(\rho) d \rho .
\end{aligned}
$$

Thus $u$ is a formal solution to $P u=0$ if $A$ satisfies the following ordinary differential equation

$$
\left(-\frac{d^{2}}{d t^{2}}+t^{2 k}\right) A(t)=\mu t^{k-1} A(t)
$$

For $u$ to be well defined in $x_{1}$ we shall require the condition

$$
A \in \mathcal{S}(\mathbb{R})
$$

Also, for $u$ to be well defined in $x_{2}$ we shall choose

$$
w(\rho)=e^{-\rho^{(k+1) / 2}} .
$$

Furthermore, the following lemma in [HH2] shows that the generalized eigenvalue problem (2.2)-(2.3) has infinitely many solutions, although only one will suffice.

Lemma 2.1. The eigenvalue problem (2.2)-(2.3) has a non-zero solution if and only if $\mu \in M$, where

$$
M=\{\mu: \mu=2 j(k+1)+k \text { or } \mu=2 j(k+1)+k+2, j=0,1,2, \cdots\} .
$$

Moreover the solution is unique up to a constant factor and is of the form

$$
A_{\mu}(t)=B_{\mu}(t) e^{-\frac{1}{k+1} t^{k+1}},
$$

where $B_{\mu}$ is a polynomial which can be computed explicitly.

Now by Lemma 2.1 and (2.4) for any $\mu \in M$ the function

$$
u_{\mu}(x)=\int_{0}^{\infty} e^{i \rho^{k+1} x_{3}+\left(\sqrt{\mu} x_{2}-1\right) \rho^{(k+1) / 2}} A_{\mu}\left(\rho x_{1}\right) d \rho,
$$

is a well defined $C^{\infty}$ function in the open set $\left\{x \in \mathbb{R}^{3}:\left|x_{2}\right|<1 / \sqrt{\mu}\right\}$, and a solution to $P u_{\mu}=0$. It remains to show that $u_{\mu}$ is not analytic near the origin. For any 
$j \in\{0,1,2, \cdots\}$ we have

$$
\begin{aligned}
\partial_{x_{3}}^{j} u_{\mu}(0) & =i^{j} A_{\mu}(0) \int_{0}^{\infty} \rho^{j(k+1)} e^{-\rho^{(k+1) / 2}} d \rho \\
& =i^{j} A_{\mu}(0)\left(2 j-1+\frac{2}{k+1}\right)\left(2 j-2+\frac{2}{k+1}\right) \cdots\left(1+\frac{2}{k+1}\right) \frac{2}{k+1} C_{0},
\end{aligned}
$$

where $C_{0}=\int_{0}^{\infty} e^{-\rho^{(k+1) / 2}} d \rho$. Here we may assume that $A_{\mu}(0) \neq 0$. Therefore

$$
\left|\partial_{x_{3}}^{j} u_{\mu}(0)\right| \geq C(2 j) !
$$

for some $C>0$ and independent of $j$. By (2.6) $u_{\mu}$ is not analytic near $0 \in \mathbb{R}^{3}$, and this completes the proof of Theorem 1.1.

\section{Final Remarks}

1. The function $A_{\mu}$ corresponding to $\mu=k$ is given by $A(t)=e^{-\frac{1}{k+1} t^{k+1}}$. Therefore by (2.5) the solution corresponding to $\mu=k$ takes the following explicit form:

$$
u(x)=\int_{0}^{\infty} e^{i \rho^{k+1} x_{3}+\left(\sqrt{k} x_{2}-1\right) \rho^{(k+1) / 2}-\frac{1}{k+1}\left(\rho x_{1}\right)^{k+1}} d \rho .
$$

2. If $k$ is an odd positive integer, and $m_{1}, \cdots, m_{\ell}$ are non-negative integers, then the operator $\Delta_{X}$ in $\mathbb{R}^{3+\ell}$ defined by

$$
\Delta_{X}=\partial_{x_{1}}^{2}+x_{1}^{k-1} \partial_{x_{2}}^{2}+x_{1}^{2 k} \partial_{x_{3}}^{2}+x_{1}^{2 m_{1}} \partial_{y_{1}}^{2}+\cdots+x_{1}^{2 m_{\ell}} \partial_{y_{\ell}}^{2}
$$

is not analytic hypoelliptic near the origin of $\mathbb{R}^{3+\ell}$, since any solution to the equation $P u=0$, with $P$ as in (1.1), is a solution to the equation $\Delta_{X} u=0$ too. If in addition $k \geq 3$, and $m_{j} \geq 1, j=1, \ldots, \ell$, then the characteristic set of $\Delta_{X}$ is equal to $\left\{x_{1}=\xi_{1}=0\right\} \subset T^{*}\left(\mathbb{R}^{3+\ell}\right)$, which is symplectic. This shows that the phenomena described above are independent of the dimension 3. For related results in the special case of dimension two see Christ [C3].

\section{REFERENCES}

[BG] M.S. Baouendi and C. Goulaouic, Nonanalytic-hypoellipticity for some degenerate elliptic operators, Bull. AMS 7878 (1972), 483-486. MR 45:5567

[C1] M. Christ, A class of hypoelliptic PDE admitting nonanalytic solutions, Contemporary Mathematics, AMS 137 (1992), 155-167. MR 93j:35048

[C2] M. Christ, A necessary condition for analytic hypoellipticity, Mathematical Research Letters 1 (1994), 241-248. MR 94m:35068

[C3] M. Christ, On local and global analytic and Gevrey hypoellipticity, Journees "Equations aux derivees partialles", Saint-Jean-de-Monts (Mai-Juin 1995). MR 96h:35029

[DZ] M. Derridj and C. Zuily, Régularité analytique et Gevrey d'opérateurs elliptiques dégénérés, J. Math. pures et appl. 52 (1973), 65-80. MR 52:11300

[GS] A. Grigis and J. Sjöstrand, Front d'onde analytique et sommes de carres de champs de vecteurs, Duke Math J. 52 (1985), 35-51. MR 86h:58136

[HH1] N. Hanges and A.A. Himonas, Singular solutions for sums of squares of vector fields, Comm. Partial Differential Equations 16 (8,9) (1991), 1503-1511. MR 92i:35031

[HH2] N. Hanges and A. A. Himonas, Singular solutions for a class of Grusin type operators, Proceedings of AMS 124, No. 5 (1996), 1549-1557. MR 96g:35035

[He] B. Helffer, Conditions necessaires d'hypoanalyticite pour des operateurs invariants a gauche homogenes sur un groupe nilpotent gradue, Journal of Differential Equations 44 (1982), 460-481. MR 84c:35026

[Ho] L. Hörmander, Hypoelliptic second order differential equations, Acta Math. 119 (1967), 174-171. MR 36:5526 
[K] J.J. Kohn, Pseudo-differential operators and hypoellipticity, Proceedings of symposia in pure mathematics XXIII (1973), 61-70. MR 49:3356

[M] G. Metivier, Une class d'operateurs non hypoelliptiques analytiques, Indiana Univ. Math J. 29 (1980), 823-860. MR 82a:35029

[OR1] O. A. Oleinik and E. V. Radkevic, The analyticity of the solutions of linear differential equations and systems, Dokladi 207, No 4 (1972), 1614-1618. MR 48:2544

[OR2] O. A. Oleinik and E. V. Radkevic, Second order equations with nonnegative characteristic form, AMS and Plenum Press, 1973. MR 56:16112

[PR] Pham The Lai and D. Robert, Sur un problem aux valeurs propres non lineaire, Israel J. of Math 36 (1980), 169-186. MR 83b:35132

[RS] L.P. Rothschild and E.M. Stein, Hypoelliptic differential operators and nilpotent groups, Acta Math 137 (1977), 247-320. MR 55:9171

[T] D. S. Tartakoff, On the local real analyticity of solutions to $\square_{b}$ and the $\bar{\partial}-$ Neumann problem, Acta Math. 145 (1980), 117-204. MR 81k:35033

[Tr] F. Treves, Analytic hypo-ellipticity of a class of pseudodifferential operators with double characteristics and applications to $\bar{\partial}$-Neumann problem, Comm. in P.D.E. 3 (1978), 475642. MR 58:11867

Lehman College, CUny, Bronx, New York 10468

E-mail address: nwhlc@cunyvm.cuny.edu

Department of Mathematics, University of Notre Dame, Notre Dame, Indiana 46556 E-mail address: alex.a.himonas.10nd.edu

Current address: Mathematical Sciences Research Institute, 1000 Centennial Drive, Berkeley, California 94720

E-mail address: himonas@msri.org 